\title{
Linear Polarization of Photons Produced by the Electron Plane Channeling in a Silicon Crystal
}

\author{
V.V. Denyak ${ }^{a}$, I.G. Evseev ${ }^{b}$, V.M. Khvastunov ${ }^{a}$, V.P. Likhachev $^{c}$, \\ S.A. Paschuk ${ }^{b}$, and H.R. Schelin ${ }^{b}$ \\ ${ }^{a}$ National Scientific Center, Kharkov Institute of Physics and Technology, Kharkov 61108, Ukraine \\ ${ }^{b}$ Federal Center of Technological Education, CEFET-PR, Curitiba, PR, CEP 80230-901, Brazil \\ ${ }^{c}$ University of São Paulo, São Paulo, Brazil
}

Received on 10 August, 2001. Revised version received on 3 October, 2002

\begin{abstract}
We present the results of the polarization and intensity measurements versus photon energy $E_{\gamma}=5-35 \mathrm{MeV}$ for the photon beam produced by the electron plane channeling with the energies 1.2 and $1.5 \mathrm{GeV}$ in silicon crystals $500 \mu \mathrm{m}$ and $290 \mu \mathrm{m}$ thick along the (110) plane. The comparison with results of another research group and theoretical calculations indicate a qualitative agreement. The correlation between the shape of the radiation intensity spectrum and its polarization energy dependence is observed.
\end{abstract}

\section{Introduction}

Recent achievements and progress of $x$-ray and gamma ray microtomography, $\mathrm{x}$-ray and gamma ray optics and their imaging properties $[1,2]$, especially in the case of material studies and non destructive tests, as well as classical studies of polarization phenomena of nuclear reactions near the threshold, which give extremely important information about the structure of nuclear matter and nuclear forces, stimulated this attempt to review the experimental methods of polarized monochromatic photon production, specifically the photon beam produced by electron channeling in crystals.

There is a relatively small number of methods of polarized photon production in the energy range of $1-100$ $\mathrm{MeV}$. Considering the physical principles they are based on, these methods could be classified as following: 1) angular selection of electron bremsstrahlung radiation; 2) Compton scattering of laser beam on ultrarelativistic electrons; 3) coherent bremsstrahlung radiation of electrons in monocrystals. Each of these methods has its own disadvantages. The first one gives very small polarization degree and not monochromatic intensity spectrum. The second method has good monochromaticity and practically $100 \%$ polarization, but low intensity and, in order to obtain with this method a beam suitable for practical work, one needs a storage ring, where electron beam frequently cross the region of interaction during the time of laser outburst. The third method gives higher intensity of photons than the others, a its quasimonochromatic spectrum has the polarization of approximately $50 \%$.

Twenty years ago the high polarization degree was found for photon beams produced by high-energy electron plane channelling in crystals [3]. Such photon beam has the inten- sity several times higher than ever produced with the mentioned above method No. 3. The maximum of this spectrum is located in the giant resonance region of atomic nuclei. These specific characteristics make such photon beam very suitable for the study of atomic nuclear structure as well as dynamic characteristics of nuclear reactions induced by polarized photons. It is clear that in this case one has to know very precisely the intensity and the polarization for the given photon beam energy. The beam intensity was actively investigated by the theoreticians and experimentalists during the last years, but the study of photon polarization has been started recently. Only few experiments were dedicated to the measurements of photon beam polarization $[4,5]$. The high polarization degree $\mathrm{P}_{\gamma}=0,6-0,9$ near the characteristic maximum was observed in these investigations. It has to be stressed that these are the unique available experimental results.

Very rare information, concerning the theoretical description of the photon polarization, was accumulated during this time. Only two research groups were involved in these investigations [4, 6]. The authors of work [4] gave two possible methods of such calculations. But, as they mentioned, their results should be considered as an illustration of some general approach. The main problem of this work is that both theoretical models are developed for the very thin crystals $(10-20 \mu \mathrm{m})$, while the experiments were carried out with the crystals of several hundred $\mu \mathrm{m}$. Of course, the comparison of such calculations and experimental results has only qualitative character.

Recently, rather interesting results were achieved in the theoretical calculation of the photon polarization on the base of the "thermal-layer" model [5] that gave very good agreement with experimental data on diamond target for electron beam initial energy $1200 \mathrm{MeV}$. At the same time, similar 
analysis [6] performed for the silicon crystal does not give a correct description of the experimental polarization spectrum of [4]. It has to be stressed that the experimental data [5] were measured at photon energies higher than in [4].

The objective of our work was the experimental investigation of the soft part of linear polarization energy spectrum of photons emitted by planar channeling of high-energy electrons in the silicon crystal. It is very important to increase the quantity of experimental information in the energy region where mentioned above disagreement was observed with the theory [6].

Moreover, it was intended to carry out simultaneously the measurements of photon intensity spectrum. Such measurements were not performed in previous investigations, although the information about the intensity spectrum is very important to test the theoretical models. The experimental conditions of the measurements have some parameters, such as initial electron beam monochromaticity, divergence, photon beam collimation etc., that should be taken into account in the computation. Since the theoretical methods for intensity spectrum calculation were developed quite well even for thick crystals, these spectra may be used as a test of accuracy of the experimental parameters analysis.

We initiated the first studies of mentioned above characteristics in the early 90's [7], but those experiments were very soon interrupted by the technical problems of the Kharkov accelerator facilities. Only recently we have got the possibilities to continue the measurements as well as to conclude the analysis of the collected data, which were preliminary presented in a previous paper [8].

\section{Experimental technique}

The experiments have been performed using the electron beam of $2 \mathrm{GeV}$ Linac of the National Scientific Center Kharkov Institute of Physics and Technology (NSC KhIPT). The experimental setup is shown in Fig.1.

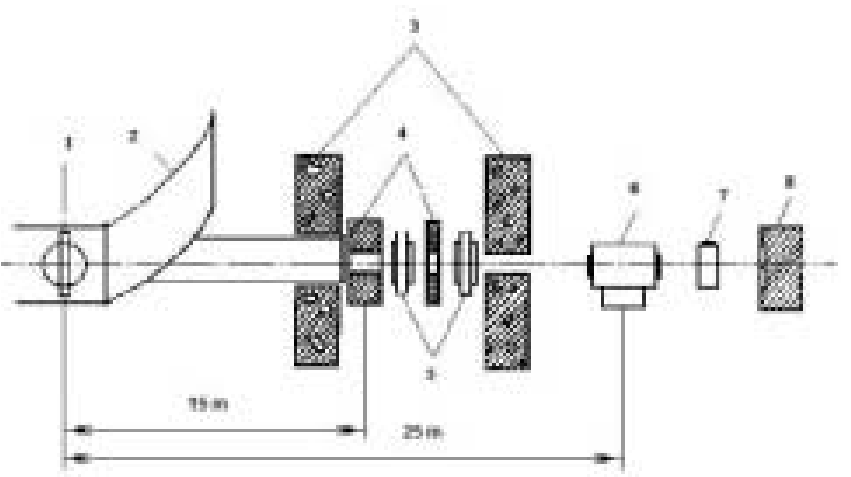

Figure 1. Experimental setup: 1 - the goniometer with the target of a silicon single crystal; 2 - the deflecting magnet; 3 - the concrete shelding; 4 - the photon beam collimator; 5 - the cleaning magnet; 6 - the deuterium polarimeter; 7 - the ionization chamber; 8 - the quantometer.

The photon beam was generated by means of a (110) plane channeling of electrons with energy $1200 \mathrm{MeV}$ and
$1500 \mathrm{MeV}$ in Si crystals $500 \mu \mathrm{m}$ and $290 \mu \mathrm{m}$ thick correspondingly. The crystal targets were orientated by a goniometer (1). The electron beam parameters at the crystal target location were: monochromaticity $\sim 1 \%$ and divergence $\sim 10^{-4}$ radian. The photon beam purity was insured by a cleaning magnet (2). The photon beam dimensions and angular divergence $\sim 10^{-4}$ radian were determined by two lead collimators (4). An additional pair of cleaning magnets (5) was installed in order to maintain the purity of the photon beam from charged particles produced by collimators. Thereafter the photon beam was directed to the experimental hall where the deuterium polarimeter (6) was installed.

The polarimeter operation [9] is based on the measurements of the proton yield asymmetry (A) of the deuterium photodisintegration, which is by definition:

$$
A=P \cdot \Sigma,
$$

Where $P$ is the photon polarization degree and $\Sigma$ is the asymmetry of the reaction $\bar{\gamma}+d=n+p$. For $E_{\gamma}=10$ $-40 \mathrm{MeV}$ the $\Sigma$-asymmetry of this reaction at $\theta=90^{\circ}$ (the secondary particles emission angle in CM system) is well known and it ranges up to $\sim 1.0$. This makes it very convenient for polarization characteristics studies of the photon beam within the mentioned energy range.

Figure 2 shows the polarimeter construction. For the proton registration, we have used the telescope of semiconductor silicon surface barrier detectors (2) which were shielded by lead (1). The solid angle of the detector telescope was determined by the dimensions of the collimator (4) and it was found to be 0.03 steradian.

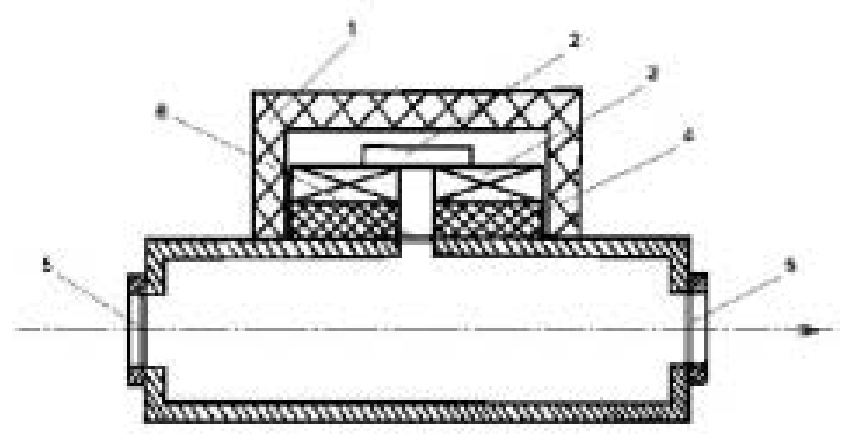

Figure 2. Polarimeter design: 1 - the lead shielding; 2 - the telescope of semiconductor detectors; 3 - the magnet; 4 - the lead collimator; 5,6 - the separating foils.

The gaseous deuterium was used as a target at the pressure varying up to $30 \mathrm{~atm}$. Low pressure was used for registration of protons with small $E_{\gamma}$. With the increase of the photon energy, the proton yield slopes down sharply due to the reaction cross section and the photon intensity decrease. To compensate this effect, high pressure for the deuterium was used. Aluminum absorbers of various thicknesses were placed in front of the detectors to extend the energy range of the registered protons. At the same time it was not possible to carry out measurements for low $E_{\gamma}$ at high pressure. 
Protons had so small energy that they were not capable to come out from the target. The construction of the polarimeter allowed us to install the detector telescope inside the deuterium chamber. Owing to this, we have registered the protons with energies down to $E_{p}=1 \mathrm{MeV}$.

The background of the measurements, mainly originating from the target and consisting of low energy electrons and positrons, presented a substantial obstacle for protons registration. It was found that the cleaning magnet (3) introduced between the collimator (4) and the detector (2) is very effective for background suppressing. The magnetic induction of the SmCo magnet used in the experiment was $0.3 \mathrm{~T}$. The deviation of charged particles in the magnetic field may be calculated with the expression:

$$
R=P /(30 \cdot H) ;
$$

where $R$ is the radius of particle motion in magnetic field (cm), $P$ is momentum transfer of particle $(\mathrm{KeV} / \mathrm{c})$ and $H$ is the magnetic induction $(T)$. The design of detecting system (i.e. collimator radius and it length, magnetic field etc.) was chosen so that the electrons and positrons with energy up to $3 \mathrm{MeV}$ were rejected from the detector area. At the same time we intend to maintain the proton trajectories with energies higher than $0.5 \mathrm{MeV}$. Such considerations decreased considerably the background of our measurements.

Besides that, electronic suppression of background was applied. When passing through the detector substance, the proton produces charged particles that create a current pulse in the preamp electrical circuit. Such pulse has a sharp front of a few nanosecond and smooth recession which is determined by the time characteristics of an integrating circuit (usually a few microseconds). The background electric pulse was produced by the huge number of electrons and positrons originated from the physical target, the vacuum elements of the experimental chamber and its constructive elements. The sum of all those small pulses (buildup) has a much longer front (approximately $0.5-1.0 \mu \mathrm{s}$ ) that is determined by the time characteristics of the accelerator beam formation. This difference in the time of pulse fronts gives a possibility to separate the proton pulses from the background pulses.

In order to detect only the fast component of the pulse, the fast preamplifier, with the entrance pulse transformer was used. Such preamplifier allows to keep the information about the primary pulse amplitude and to suppress the background more than ten times. The characteristic duration of a pulse in our experiments was $5-10 \mathrm{~ns}$, but it depends on various experimental conditions at each experiment, at the LINAC of NSC KhIPT [10].

The crystal orientation was determined by measuring the intensity of the low energy photons with the ionization chamber (7) (see Fig. 1). The orientation measurement accuracy was $5 \cdot 10^{-5}$ radian. The crystal was positioned in such a way that the beam axis was close to the crystal $<111>$ axis. Fig. 3 shows the typical intensity orientation dependence and the relative position of the crystal planes. The location of the three largest peaks correspond to the

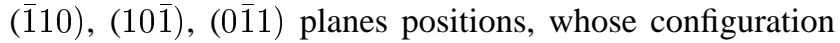
coincides with the (110) crystal plane. Two smaller peaks originate from three $(11 \overline{2}),(\overline{2} 11),(1 \overline{2} 1)$ planes and others maxima reflect the location of less pronounced planes.

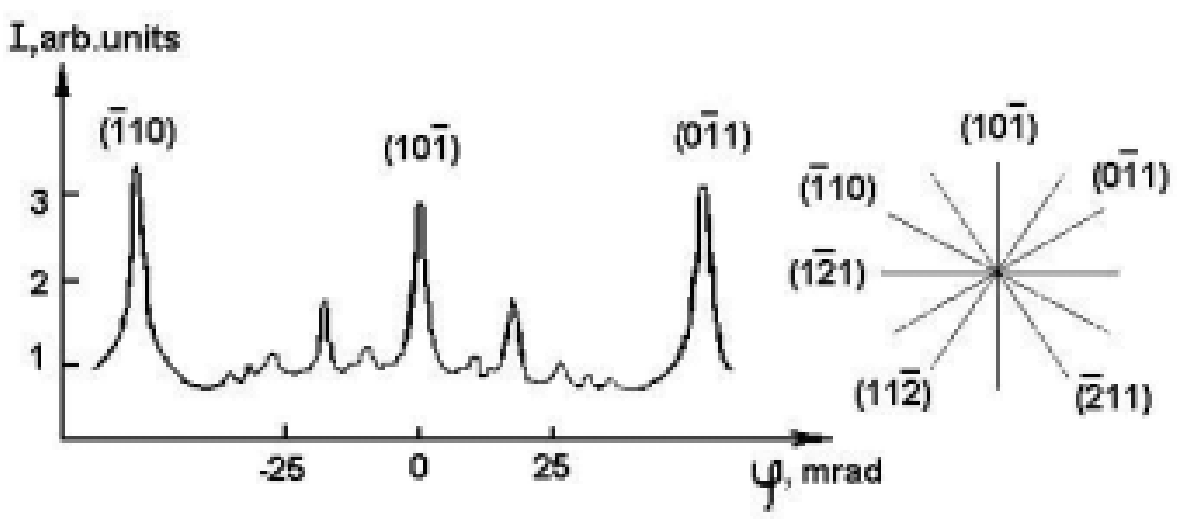

Figure 3. Characteristic orientation dependence of low-energy photon yield and the crystal planes map.

The polarization vector direction has been changed by the crystal plane rotation by $90^{\circ}$ around the beam axis. The photon spectra, corresponding to the amorphous target, were measured with the same crystal by deviating it from the plane by a small angle, where no peaks of intensity, from any plane, were observed.

In order to determine the possible proton yield asymme- try caused by the experimental device asymmetry, we have measured the proton yield at two positions of the disoriented crystal. They are distinguished by the crystal rotation at $90^{\circ}$ around the beam axis. The comparison of these measurements does not indicate the instrument asymmetry within the limits of the experimental error.

The photon beam monitoring was made with the quan- 
tometer (8) (See Fig. 1). Simultaneously, by measuring the emission current from the crystal, we controlled the electron intensity that passed through the target. The difference in the emission current for the orientated and disoriented crystal does not exceed 3\% and it was included in the data processing.

\section{Experimental results}

As a result of the measurements, the proton energy spectra for three different crystal orientations were obtained. They correspond to three directions of the photon polarization vector: the parallel $\left(N_{\|}\right)$and perpendicular $\left(N_{\perp}\right)$ to the reaction plane as well as for the disoriented crystal $\left(N_{o}\right)$. The registered protons were considered as a result of direct photodisintegration of the deuteron. Thus, we were able to restore the photon energy spectra according to the reaction kinematics. The possible contribution of protons generated at $E_{\gamma}$ above the meson production threshold is small, mainly due to the slope character of the photon intensity spectra $\left(\sim 1 / E_{\gamma}\right)$ and it does not exceed a few percents.

The photon beam polarization had been restored according to the formula:

$$
P_{\gamma}=A / \Sigma=\left(N_{\|}-N_{\perp}\right) /\left(\Sigma\left(N_{\|}+N_{\perp}\right)\right)
$$

For the photon energy region above $10 \mathrm{MeV}\left(E_{\gamma}>10\right.$ $\mathrm{MeV}$ ) the deuteron photodisintegration asymmetry $(\Sigma)$ values were taken from the experimental data [11]. For lower energies $E_{\gamma}$, we used the results of the reaction asymmetry calculations based on the covariant gauge-invariant model with a Paris potential [12]. In this work, the interaction in the final state was considered in the form of re-scattering in the ${ }^{1} \mathrm{~S}_{0}$ state, and it was concluded that deuteron photodisintegration asymmetry within the range $E_{\gamma}=4-10 \mathrm{MeV}$ is constant and equal to 0.99 .

Our results of beam polarization are shown in Fig. 4 together with experimental data [4] obtained under conditions very close to our measurements (silicon crystal 400 $\mu \mathrm{m}$ thick, (110) plane, and electron beam with the energy $900 \mathrm{MeV})$.

The upper part of Fig. 4 shows the radiation intensity extracted from our experimental data on the oriented crystal and normalized by the disoriented crystal case as following:

$$
\beta=\left(N_{\|}+N_{\perp}\right) / 2 N_{0}
$$

In spite of the fact that polarization measurements of [4] were carried out with another experimental technique (Compton polarimeter), they are in qualitative agreement with our measurements at initial electron energy $E_{e}=1200$ $\mathrm{MeV}$. However one can see that our experiment gave larger polarization degree than could be attributed to higher $E_{e}$ leading to the shift of the radiation maximum to higher $E_{\gamma}$ $\left(\sim E_{e}^{3 / 2}\right)$.
Comparison of our results for $E_{e}=1200 \mathrm{MeV}$ with theoretical calculations from work [4] (Fig. 4c) shows that modeling gives the description of polarization energy dependence more correct than the continuous model. Such dependence should have a broad maximum at $E_{\gamma}=5-10$ $\mathrm{MeV}$ with a flattening out at $E_{\gamma}=20-30 \mathrm{MeV}$.

At Fig. 4a the results from theoretical calculations on the base of "thermal-layer" model are shown. Despite the fact that the authors performed this analysis using the parameters very close to ours for electron beam initial energy $1200 \mathrm{MeV}$, their calculations agree with our experimental data only qualitatively. As it is seen from the intensity, this calculation overestimated the coherent effect (i.e. theoretical intensity spectrum is higher than the experimental). This, of course, leads to higher polarization, since the polarization value should depend on the number of electrons involved in channeling motion (i.e. in coherent radiation).

Comparing the polarization for $E_{e}=900 \mathrm{MeV}, E_{e}=1200$ $\mathrm{MeV}$ and $E_{e}=1500 \mathrm{MeV}$ one can see that the argument of initial electron energy leads to the increase of the polarization. Such effect was not observed earlier for diamond crystal [5] due to a lack of experimental accuracy. However, the theoretical predictions on the base of the "thermal-layer" model, in the same work, show such polarization dependence from initial electron energy also for diamond crystal. From the intensity spectra for $E_{e}=1200 \mathrm{MeV}$ and $E_{e}=1500$ $\mathrm{MeV}$ one can see that the increase of the electron energy leads to bigger coherent effect and this causes the increase of the polarization.

From Fig. 4 ( $a$ and $b$ ) it is seen that the characteristic feature of channeling radiation is that the beam polarization energy dependence is very similar to the intensity energy dependence. This prompts an idea that the radiation is formed by the sum of the coherent part, produced by the electrons involved in motion in the plane channel, and by the bremsstrahlung part, produced by electrons not involved in the channeling process. The coherent part should have the $100 \%$ polarization that is constant in the whole energy region. To check this suggestion, the polarization in the coherent part of the spectrum was calculated from the experimental data according to the formula:

$$
P_{c}=\left(\left(N_{\|}-N_{0}\right)-\left(N_{\perp}-N_{0}\right)\right) /\left(\left(\left(N_{\|}-N_{0}\right)+\left(N_{\perp}-N_{0}\right)\right) \Sigma\right)
$$

The results of these calculations are given in Fig. 5. It is seen that polarization in the coherent part of the spectrum is almost constant. Their values are very close to $100 \%$ but are not equivalent. This means that the idea mentioned above is very truthful but is not completely correct. The correlation between intensity energy dependence and polarization energy dependence exist but the coherent part of the radiation is not completely polarized. 
Comparison of Fig. 5a and Fig. 5b shows that the increase of initial electron energy results in higher polarization of the coherent radiation. It means that the increase of the total photon beam polarization with the electron energy can not be explained only by a larger number of electrons involved in channeling process. There should be another mechanism that has rather large influence on the process of polarized photons production by channeling electrons.
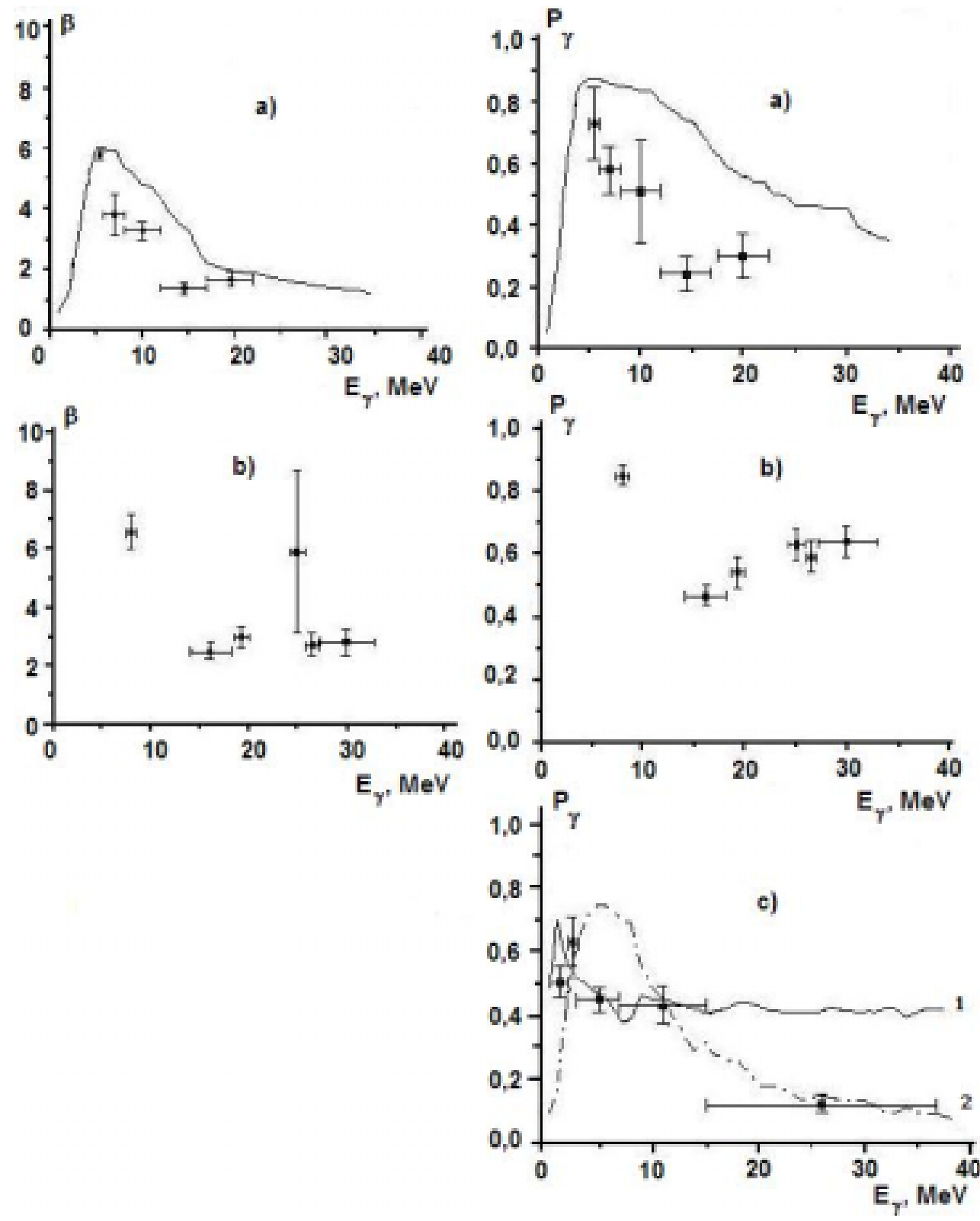

Figure 4. Relative intensity and polarization degree of the photon beam. a) $E_{e}=1200 \mathrm{MeV}, t=500 \mu \mathrm{m}$ curves - results of work [6]; b) $E_{e}=$ $1500 \mathrm{MeV}, t=290 \mu \mathrm{m} ; \mathrm{c}$ ) data from paper [4]: $E_{e}=900 \mathrm{MeV}, t=400 \mu \mathrm{m}$ - modeling and (2) - continuous model.

\section{Conclusions}

As a result of the measurements, the proton energy spectra for three different crystal orientations were obtained. They correspond to three directions of the photon polarization vector: the parallel $\left(N_{\|}\right)$and the perpendicular $\left(N_{\perp}\right)$ to the reaction plane as well as for the disoriented crystal $\left(N_{0}\right)$. The registered protons were considered as a result of direct photodisintegration of the deuteron. Thus, we were able to restore the photon energy spectra from the reaction kinematics. The possible contribution of protons generated at $E_{\gamma}$ above the meson production threshold is small mainly due to the slope character of the photon intensity spectra $\left(\sim 1 / E_{\gamma}\right)$ and it does not exceed a few percents.

Investigated low energy part of photon beam spectrum, produced by planar electron channeling in silicon crystal, possesses the peak with the high polarization degree $\sim 80 \%$ and its intensity exceeds approximately in $5-6$ times the 
amorphous level. The comparison with the "thermal-layer" model calculations shows that it overestimates the polariza

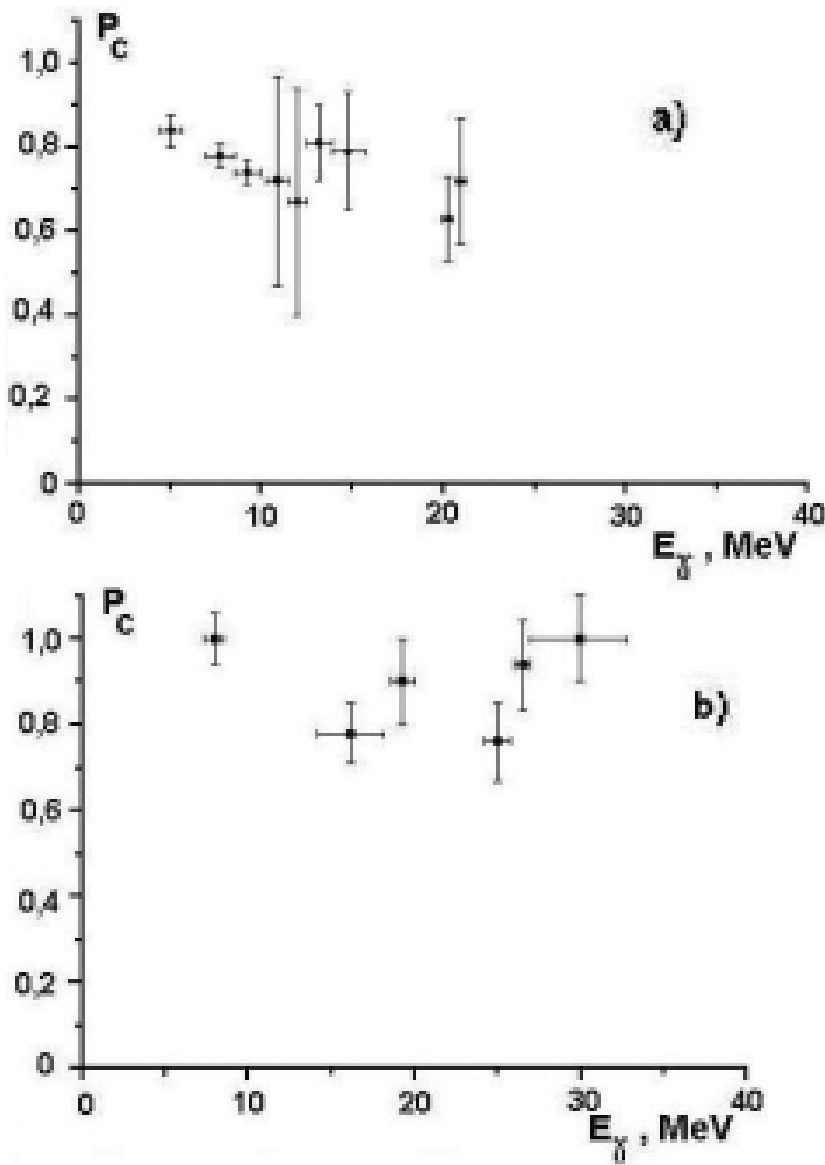

Figure 5. Polarization in the coherent part of the spectrum. a) $E_{e}=$ $1200 \mathrm{MeV}, \mathrm{t}=500 \mu \mathrm{m}$; b) $E_{e}=1500 \mathrm{MeV}, \mathrm{t}=290 \mu \mathrm{m}$.

tion for silicon crystal despite good theoretical description of the experimental results on diamond. It was found that the characteristic feature of the channeling radiation is that the beam polarization, as a function of photon energy, reflects the behavior of the radiation intensity spectrum itself. The polarization of the coherent part of radiation doesn't depend on the photon energy and reaches almost $100 \%$. The increase of the initial electron energy results in polarization enlargement both for the total photon beam and for the coherent radiation.

From previous investigations it is known that the energy position of the intensity maximum is a function of initial electron energy $\sim E_{e}^{3 / 2}$. Thus, varying the initial electron energy within the limits $1-2 \mathrm{GeV}$, one can obtain the peak maximum at $E_{\gamma}=5-15 \mathrm{MeV}$ with the spectrum approaching the amorphous level at $E_{\gamma}=40-50 \mathrm{MeV}$, i.e., in the range of the thresholds of many photonuclear reactions and giant resonances as well. This energy region is extremely interesting considering that here one can achieve a low level of random coincidences when performing the coincidence photo initiated experiments on studying polarization characteristics of two-particle photonuclear reactions [13]. From this point of view, it will be very interesting to carry out the systematic experimental and theoretical investigations of polarization energy dependence from different crystal materials, their thickness and initial electron energy.

\section{Acknowledgements}

The authors are very thankful to Kasilov V.I., Sanin V.M, and Shcherbak S.F. for their significant help in photon beam production using the electron beam of $2 \mathrm{GeV}$ Linac of the National Scientific Center Kharkov Institute of Physics and Technology.

This work was partially supported by CNPq/RHAE and the authors are very thankful for these Brazilian Governmental Agencies. It has to be mentioned the support and productive discussions we had with our colleagues from Federal Center of Technological Education (CEFET-PR) as well as our colleagues from the Physics Institute of the Sao Paulo University, which gave us the possibility to conclude this work.

\section{References}

[1] R. Fitzgerald, Phase-Sensitive X-Ray Imaging, Phys. Today, April of 1999.

[2] R.D. Spal, Submicrometer Resolution Hard X-Ray Holography with the Asymmetric brag Diffraction Microscope, Phys. Rev. Lett. 86, 3044 (2001)

[3] Yu.N. Adishchev, I.E. Vnukov, S.A. Vorobev, V.M. Golovkov, V.N. Zabaev, V.I. Lunev, A.A. Kurkov, JETP Letters 33, 462 (1981).

[4] S.A. Vorobyov, E.G. Vyatkin, Yu.L. Pivovarov, A.P. Potylitsyn, and I. Khakberdiyev, J. of Exp. and Theor. Phys. 94, 38 (1988).

[5] V.B. Ganenko, V.A. Gushchin, Yu. V. Zhebrovskiy, L.Yu. Kolesnikov, I.N. Mondrus, N.N. Nasonov, and V.D. Ovchinnik, Phys. of Atom. Nuclei, 60, 165 (1997).

[6] I.N. Mondrus, N.N. Nasonov, Poverkhnost', Fizika, Khimiya, Mekhanika. In Russian, 5, 76 (1995).

[7] V.V. Denyak, I.G. Evseev, L.M. Zavada, V.I. Kasilov, S.V. Kas'yan, N.I. Lapin, V.P. Likhachev, V.I. Noga, S.A. Pashchuk, V.M. Sanin, V.M. Khvastunov. Proceeding of XXI All Union Workshop on Physics of Charged Particles Interaction with Crystals. Moscow: Moscow University, 76 (1991).

[8] V.V. Denyak, S.A. Paschuk, I. Evseev, H.R. Schelin. Linear Polarization of Photons Produced by the Electron Plane Channeling in a Silicon Crystal. In: XXIII Reunião de Trabalho sobre Física Nuclear no Brasil, 2000, Campinas. Programa e Resumos de XXIII Reunião de Trabalho sobre Física Nuclear no Brasil. São Paulo, SP: Sociedade Brasileira de Física, 2000.

[9] Yu.V.Vladimirov, V.V. Denyak, I.G. Evseev, V.I. Kasilov, N.I. Lapin, V.P. Likhachev, S.A. Pashchuk, E.V. Pegushin, G.A. Savitskii, V.M. Sanin, V.M. Khvastunov, S.F. Shcherbak, Preprint KhIPT, 88-60 (1988). 
[10] V.P. Likhachev, Yu.V.Vladimirov, I.G. Evseev, S.A. Pashchuk, G.A. Savitskij, V.I. Trotsenko, V.B. Shostak, and V.M. Tkach, Preprint KhIPT, 85-21 (1985).

[11] W.Del Bianko, L. Federici, G. Giordano G. Matone, G. Pasquariello, P. Picozza, R. Caloi, L.Casano, M.P. De Paskale, L. Ingrosso, M. Mattioli, E. Poldi, C. Schaerf, P. Pelfer, D. Prosperi, Phys. Rev. Lett. 47, 1118 (1981).

[12] A.Yu. Buki, Yu.V. Vladimirov, V.V. Denyak, I.G. Evseev,
A.A. Zayats, Yu.A. Kasatkin, V.I. Kasilov, I.K. Kirichenko, N.I. Lapin, V.P. Likhachev, S.I. Nagornyi, S.A. Pashchuk, E.V. Pegushin, V.M. Sanin, V.M. Khvastunov, S.F. Shcherbak. Sov. J. of Nucl. Phys. 51, 769 (1990).

[13] N.A.Burkova, V.V. Denyak, R.A. Ehramzhyan, I.G. Evseev, V.M. Khvastunov, V.P. Likhachev, S.A. Pashchuk, and M.A. Zhusupov, Nucl. Phys. A 586, 293 (1995). 